\title{
Auszug aus einem Schreiben an den Herausgeber.
}

\author{
(Von Herrn H. Schubert in Potsdam.)
}

...... Herr Zeuthen in Copenhagen hat die Güte gehabt, einige von ihm verfasste, die Bestimmungen von Charakteristiken betreffende $A b-$ handlungen durch Ihre Vermittelung in meine Hände gelangen zu lassen. Unter denselben befindet sich eine mir bisher unbekannt gebliebene in dänischer Sprache geschriebene Arbeit „Bestemmelse af Charakteristikerne i de elementaere Systemer af Flader af anden Orden"6, deren Inhalt mich zur Berichtigung einer Annahme veranlasst, die ich bei der Abfassung meiner im $71^{\text {ten }}$ Bande Ihres Journals veröffentlichten, ,Zur Theorie der Charakteristiken"6 betitelten Arbeit gemacht habe. In meiner aus dem Studium der Abhandlungen des Herrn Chasles in den Comptes rendus der Pariser Akademie über diesen Gegenstand hervorgegangenen Arbeit ist nämlich der von Herrn Chasles ausgesprochene Gedanke ausgeführt, die Elementarcharakteristiken der Flächen zweiter Ordnung aus den Zahlen zu bestimmen, welche angeben, wieviel degenerirte Flächen in einem Elementarsysteme vorkommen. Diese von mir mit $\sigma, \varepsilon, \varkappa$ bezeichneten Zahlen sind in meiner Arbeit unter der Voraussetzung behandelt, dass bis dahin weder eine Werthangabe noch eine ausführliche Begründung derselben publicirt worden sei. In dem ersten Theile dieser Annahme habe ich mich aber, wie ich jetzt sehe, getäuscht. Denn der oben èrwähnten Abhandlung des Herrn Zeuthen ist eine "Oversigt" beigefügt, in welcher nicht bloss die auch von Herrn Chasles angegebenen Elementarcharakteristiken $\mu, \nu, \varrho$, sondern auch Gleichungen angeführt werden, aus denen die Werthe der Zahlen $\sigma, \varepsilon, *$ ersichtlich sind. Ich will nicht unterlassen, die für diesen Punkt Herrn Zeuthen gebührende Priorität hiermit anzuerkennen.

Potsdam, den 3. December 1870. 